\title{
Effect of Chemical Oxidation on the Self-Assembly of Organometallic Block Copolymers
}

\author{
Hany B. Eitouni, ${ }^{1,2}$ Nitash P. Balsara, ${ }^{1,2,3 *}$ \\ ${ }^{1}$ Department of Chemical Engineering, ${ }^{2}$ Materials Sciences Division, and ${ }^{3}$ Environmental \\ Energies and Technologies Division, Lawrence Berkeley National Laboratory, University \\ of California, Berkeley, California 94720
}

\section{Supporting Information}

Synthesis, Chemical Oxidation, and Molecular Characterization of SF and IF polymers

The poly(styrene-block-ferrocenyldimethylsilane) copolymers and the poly(isoprene-block-ferrocenyldimethylsilane) copolymers are synthesized under high vacuum in benzene, using $s$-butyllithium as the initiator and isopropanol as the terminator. For each copolymer the polystyrene (S) block or the polyisoprene (I) block is synthesized first followed by the poly(ferrocenyldimethylsilane) (F) block by anionic and anionic ring opening polymerization, respectively. The polydispersity indices of the SF and IF copolymers are 1.03 and 1.01, respectively. Additional details are given in ref. 16 .

Solutions of the block copolymers in degassed benzene and silver nitrate in degassed methanol were mixed in an argon filled glovebox and stirred for 12 hours. Upon mixing, the orange-yellow color of the SF and IF polymer solutions changed instantaneously to green. This is a classic signature of oxidation of ferrocene to ferrocenium. The oxidized solutions were centrifuged and the supernatant was passed through $0.2 \mu \mathrm{m}$ filters to remove silver aggregates. Samples were then freeze-dried from methanol-benzene solutions. 


\section{UV-Visible Spectroscopy}

The spectra were obtained on a Cary 400Bio UV-visible spectrometer. Samples were prepared as $0.1 \mathrm{wt} \%$ solutions of polymer in benzene. The spectra for the SF series are shown in Figure S-1. The data are very similar to the IF series shown in Figure 1.

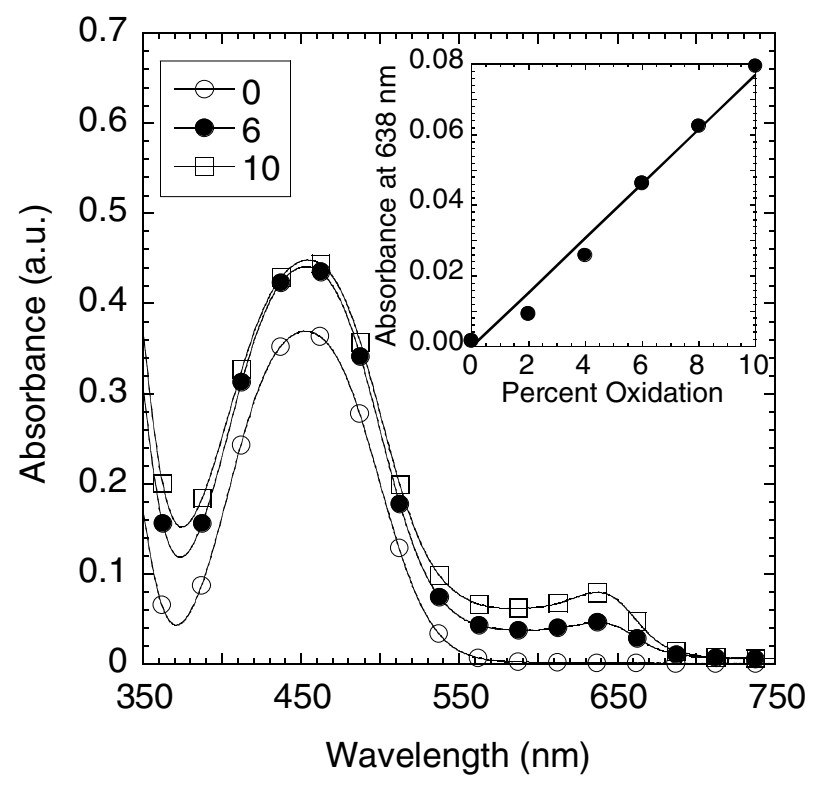

Figure S-1. UV-Visible spectra of the SF copolymers for different oxidation percentages $(100 x)$. The inset shows the absorbance at $638 \mathrm{~nm}$, the wavelength of the ferrocenium cation absorption peak, versus percent oxidation.

\section{SAXS Instrumentation}

The SAXS data presented here were performed on a custom-built instrument in the Materials Research Laboratory at the University of California, Santa Barbara (UCSB). The instrument was configured with X-rays of wavelength $\lambda=0.154 \mathrm{~nm}(\Delta \lambda / \lambda \sim$ $0.1 \%$ ) focused independently in the horizontal and vertical planes to give a beam size of $\sim 1 \mathrm{~mm}$ square. The samples were held in sealed brass sample cells with Kapton windows. Two-dimensional X-ray scattering patterns were collected on a multiwire X-ray area 
detector (Bruker HI-STAR) with an $11 \mathrm{~mm}$ diameter. The two dimensional scattering patterns were azimuthally averaged. We report the SAXS intensity I as a function of scattering vector $\mathrm{q}(\mathrm{q}=4 \pi \sin (\theta / 2) / \lambda$ where $\theta$ is the scattering angle). The SAXS experiments were repeated many times on separate batches of oxidized SF and IF samples to ensure reproducibility. All of the data presented here were taken during a single 4-day run using the same sample oven and instrument configuration.

\section{SAXS Results}

The SAXS intensity profiles were fit to the Gaussian form given in the text for each temperature. $\mathrm{T}_{\mathrm{ODT}}$ was evidenced as either a discontinuity in the plot of peak width versus temperature or as a change in the slope of the plot. There was also a discontinuity observed in peak intensity at the $\mathrm{T}_{\mathrm{ODT}}$ as seen in Figure $\mathrm{S}-2$ for the $\mathrm{SF} x=0.04$ sample.

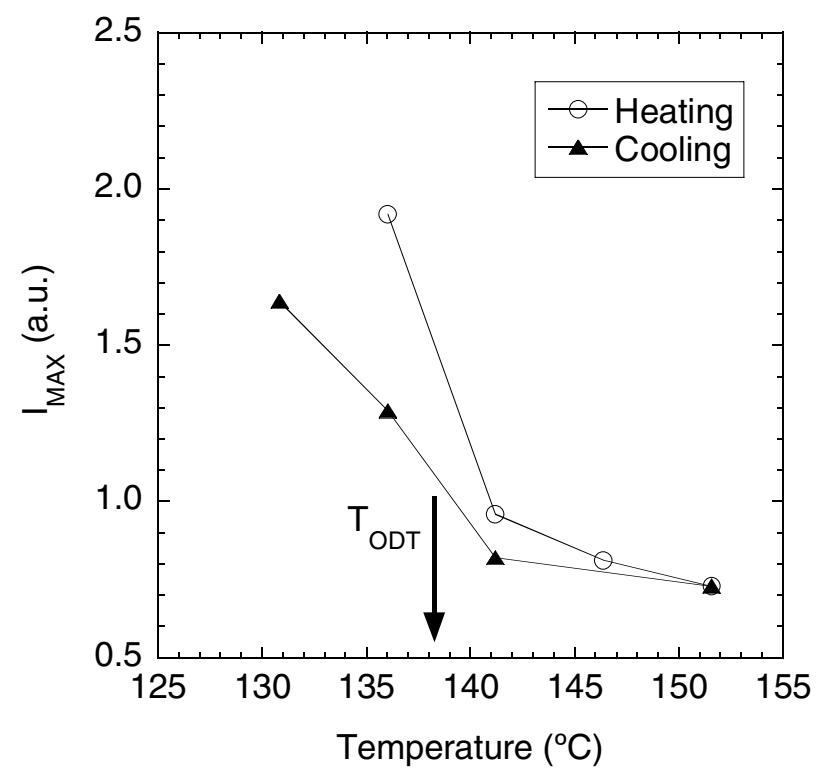

Figure S-2. Peak intensity, $\mathrm{I}_{\mathrm{MAX}}$, of the SF sample with $x=0.04$ as a function of temperature for both heating and cooling of the sample. 
We expect lamellar order in the SF and IF copolymers due to their symmetric composition. This was confirmed for the SF case $\left(\phi_{\mathrm{F}}=0.56\right)$ by determining the location of the second order SAXS peak as reported in Ref. 16. However, we were unable to detect the second order peak in the SAXS profiles obtained from the IF copolymer $\left(\phi_{\mathrm{F}}=0.48\right)$ in the weakly ordered state. As the block copolymer becomes more symmetric $(\phi \rightarrow 0.5)$, the second order SAXS peak becomes increasingly difficult to observe due to an overlap with the minimum in the form factor of lamellae at the same q value. Higher order scattering peaks are also affected by the limited long-range order in weakly ordered block copolymers, and the fact that the composition profiles in the ordered state become increasingly sinusoidal. ${ }^{\mathrm{S}} \quad$ Determination of structure in nearly symmetric block copolymers is thus often based on the analysis of the primary peak alone (e.g. ref. S1).

\section{Stability of Oxidized Chains}

Care was taken to ensure that the ferrocenium-containing polymers did not undergo degradation due to the presence of oxygen. The samples were prepared and handled in an Argon glove box until in their solvent-free state. We found that ferrocenium-containing polymers were prone to degradation in the solution state but not in the dried state if exposed to air.

Chain degradation will lead to a decrease in $\mathrm{R}_{\mathrm{g}}$, the radius of gyration of the chains. The location of the SAXS peak in $\mathrm{q}$-space ( $\mathrm{q}_{\text {peak }}$ ) provides a measure of the size of the chains in the melt. ${ }^{\mathrm{S} 1}$ Figure S-3 shows the dependence of $\mathrm{q}_{\text {peak }}$ on $x$ for both SF and IF systems at $146^{\circ} \mathrm{C}$. Using the theory of Leibler, ${ }^{\mathrm{S} 2}$ we obtain that $\mathrm{R}_{\mathrm{g}}$ of both the SF 
and IF copolymers is $5.6 \pm 0.2 \mathrm{~nm}$. The fact that $\mathrm{R}_{\mathrm{g}}$ is independent of $x$ is an indication that chemical oxidation does not lead to chain degradation.

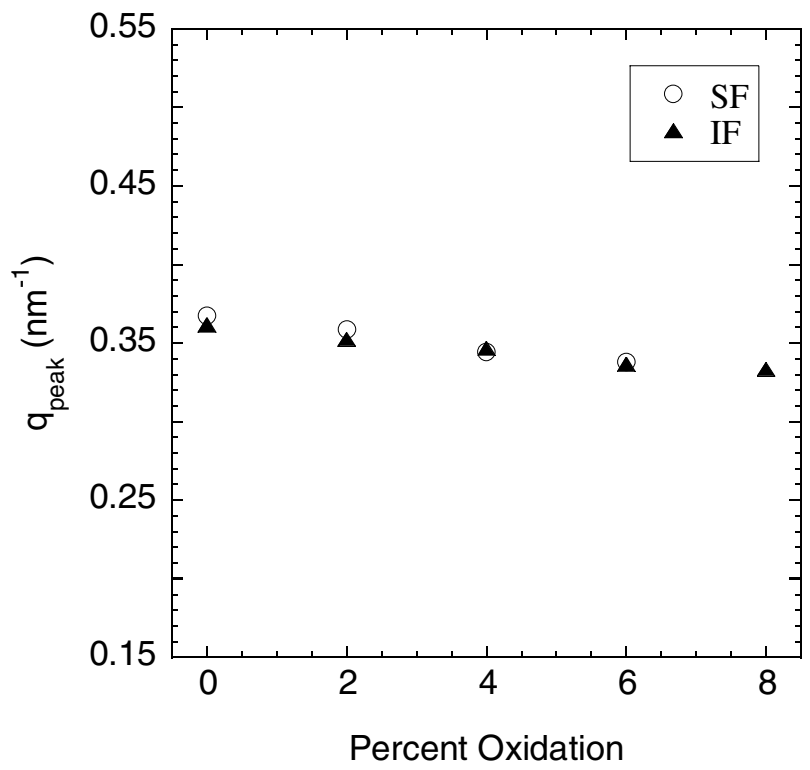

Figure S-3. The location of the SAXS intensity peak, $\mathrm{q}_{\text {peak }}$, for both the SF and IF copolymers as a function of oxidation percentage $(100 x)$ at $146^{\circ} \mathrm{C}$.

Gel permeation chromatography (GPC) with both viscosity and light scattering detection was also used to confirm that the polymers did not degrade after oxidation. GPC was run on an SF polymer not used in this study but that was characterized in Ref. 16. The polymer has a total molecular weight of $21 \mathrm{~kg} / \mathrm{mol}$ and a volume fraction of the F block of 0.34. Oxidized samples were prepared as described above and benzene was used as the eluent in the GPC. GPC traces using the light scattering detector are shown for the unoxidized and oxidized samples in Figure S-4 (similar results were obtained using the viscosity detector). The elution volume and the profiles for the unoxidized and oxidized samples are nearly identical. These results support our claim that our chemical oxidation procedure does not lead to chain degradation. The GPC and SAXS results 
(Figures S-3 and S-4) together indicate that oxidation has no effect on chain dimensions in both melts as well as solutions.

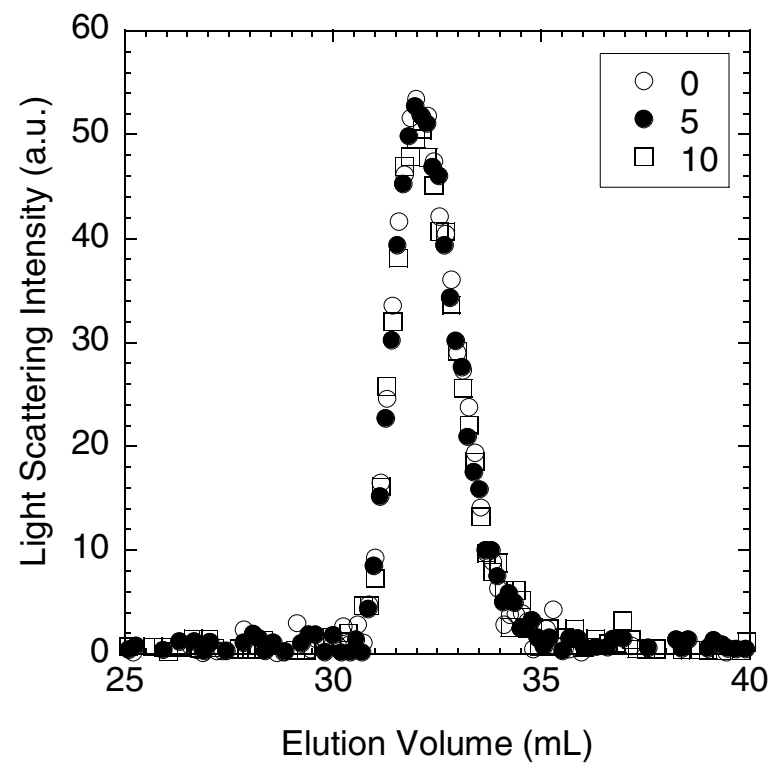

Figure S-4. GPC traces for an SF polymer that has a total molecular weight of $21 \mathrm{~kg} / \mathrm{mol}$ and a volume fraction of the F block of 0.34 for different oxidation percentages $(100 x)$.

\section{Calculation of Dissociation Constant}

Based on the dielectric constant of polystyrene (2.5) and poly(ferrocenyldimethylsilane) ${ }^{14}(2.8)$, the ratio of the Bjerrum length to the monomer size, $\mathrm{u}$, was determined to be 31 at $150{ }^{\circ} \mathrm{C}\left(\mathrm{u}=\mathrm{e}^{2} / 6 \varepsilon \varepsilon_{\mathrm{o}} \mathrm{akT}\right.$ where e is the elementary charge, $\varepsilon$ is the dielectric constant, $\varepsilon_{0}$ is the permittivity of free space, and a is the average statistical segment length which was assumed to be $10 \AA$ ). Using this constant and the volume fraction of the F block in our SF copolymer (0.56), the structure factor given in ref. 15 allowed the calculation of $\chi_{\mathrm{ODT}} \mathrm{N}$ as a function of number of charges per chain, where $\chi_{\mathrm{ODT}}$ is the Flory-Huggins interaction parameter at the order-disorder transition 
and $\mathrm{N}$ is the number of monomers per chain. From the experimentally determined $\mathrm{T}_{\mathrm{ODT}}$ 's for our samples and the known temperature dependence of $\chi$ for the SF system, ${ }^{16}$ we calculated $\chi_{\mathrm{ODT}} \mathrm{N}$ for the SF samples with $x=0$ and 0.08 to be 11.08 and 11.12, respectively, assuming $\chi$ is unaffected by the oxidation process. By comparing the ratio of these two $\chi_{\mathrm{ODT}} \mathrm{N}$ 's to the zero charge $\chi_{\mathrm{ODT}} \mathrm{N}$ from the structure factor calculation (10.79), we determined the number of dissociated counter ions in the $x=0.08$ sample to be 0.064 dissociated charges per chain. Based on the molecular weight of the F block (13.8 $\mathrm{kg} / \mathrm{mol}$ ) and $x=0.08$, we expect the number of ferrocenium nitrate pairs to be 4.6 per chain. This leads to an estimated dissociation constant of 0.014 . When this analysis is performed over the studied oxidation range, $x=0.02$ to 0.08 , the average dissociation constant is determined to be 0.019 , which is reasonable considering the low dielectric constant of our polymers.

An analysis of the IF system yields similar results. The experimentally determined temperature dependence of the $\chi$ parameter for the IF system is given by

$$
\chi=0.023+5.42 / \mathrm{T}
$$

where $\mathrm{T}$ is expressed in Kelvin. Using a similar analysis to that used for the SF system, $\mathrm{u}$ was calculated to be 32 . Using this constant and the volume fraction of the F block in our IF copolymer ( 0.48$)$, the structure factor given in ref. 15 allowed the calculation of $\chi_{\mathrm{ODT}} \mathrm{N}$ as a function of number of charges per chain. Based on the experimentally determined $\mathrm{T}_{\mathrm{ODT}}$ 's for the IF samples and the temperature dependence of $\chi$ for the IF system given in equation 1 , we calculated $\chi_{\mathrm{ODT}} \mathrm{N}$ for the IF samples with $x=0$ and 0.08 to be 10.57 and 10.68, respectively, again assuming $\chi$ is unaffected by the oxidation process. As 
performed above, by comparing the ratio of these two $\chi_{\mathrm{ODT}} \mathrm{N}$ 's to the zero charge $\chi_{\mathrm{ODT}} \mathrm{N}$ from the structure factor calculation (10.53), we determined the number of dissociated counter ions in the $x=0.08$ sample to be 0.10 dissociated charges per chain. Based on the molecular weight of the $\mathrm{F}$ block $(9.8 \mathrm{~kg} / \mathrm{mol})$ and $x=0.08$, we expect the number of ferrocenium nitrate pairs to be 3.2 per chain. This leads to an apparent dissociation constant of 0.032 . Again, when this analysis is performed over the studied oxidation range, $x=0.02$ to 0.08 , the average dissociation constant is determined to be 0.029 , which is in reasonable agreement with the results of the analysis of the SF system.

\section{References}

(S1) Rosedale, J.H.; Bates, F.S.; Almdal, K.; Mortensen, K.; Wignall, G.D.

Macromolecules 1995, 28, 1429-1443.

(S2) Leibler, L. Macromolecules 1980, 13, 1602-1617. 